\title{
Involvement of Nav 1.8 sodium ion channels in the transduction of mechanical pain in a rodent model of osteoarthritis
}

\author{
Niklas Schuelert ${ }^{1}$ and Jason J McDougall ${ }^{1,2^{*}}$
}

\begin{abstract}
Introduction: A subgroup of voltage gated sodium channels including Nav1.8 are exclusively expressed on small diameter primary afferent neurons and are therefore believed to be integral to the neurotransmission of nociceptive pain. The present study examined whether local application of A-803467, a selective blocker of the Nav 1.8 sodium channel, can reduce nociceptive transmission from the joint in a rodent model of osteoarthritis (OA).

Methods: OA-like changes were induced in male Wistar rats by an intra-articular injection of $3 \mathrm{mg}$ sodium monoiodoacetate (MIA). Joint nociception was measured at day 14 by recording electrophysiologically from knee joint primary afferents in response to non-noxious and noxious rotation of the joint both before and following close intra-arterial injection of A-803467. The effect of Nav1.8 blockade on joint pain perception and secondary allodynia were determined in MIA treated animals by hindlimb incapacitance and von Frey hair algesiometry respectively.

Results: A-803467 significantly reduced the firing rate of joint afferents during noxious rotation of the joint but had no effect during non-noxious rotation. In the pain studies, peripheral injection of A-803467 into OA knees attenuated hindlimb incapacitance and secondary allodynia.

Conclusions: These studies show for the first time that the Nav1.8 sodium channel is part of the molecular machinery involved in mechanotransduction of joint pain. Targeting the Nav1.8 sodium channel on joint nociceptors could therefore be useful for the treatment of OA pain, avoiding the unwanted side effects of nonselective nerve blocks.
\end{abstract}

\section{Introduction}

Osteoarthritis (OA) is a musculoskeletal disorder in which joint degeneration leads to a loss of mobility and function. OA primarily affects the weight bearing joints (for example knees, hips) and is typified by synovitis and degeneration of the articular cartilage and subchondral bone. The most prominent feature but least understood aspect of OA is joint pain which typically worsens with weight bearing and activity. The clinical diagnosis and treatment of OA pain has proven to be a difficult challenge because of the multitude of complex underlying mechanisms and the fact that different patients show a varied response to the same therapy. The preferred first

\footnotetext{
* Correspondence: jason.mcdougall@dal.ca

'Department of Physiology \& Pharmacology, University of Calgary, 3330,

Hospital Drive NW, Calgary, AB, T2N 4N1, Canada

Full list of author information is available at the end of the article
}

line treatment for OA pain is non-steroidal anti-inflammatory drugs; however, the beneficial outcome of these drugs is limited and some patients fail to achieve any pain relief at all. Therefore, there is a pressing need to understand the causes and mechanisms of OA pain so that more efficacious targets can be identified to help alleviate these debilitating symptoms. Chronic joint pain originates in the periphery by the sensitization of primary afferent nerve fibers innervating the joint leading to heightened neuronal activity [1-4]. Prolonged bombardment from these primary afferents subsequently sensitizes spinal and supra spinal neurons leading to the experience of persistent pathological pain.

Intra-articular injection of monosodium iodoacetate (MIA) inhibits glyceraldehye-3-phosphate dehydrogenase activity in chondrocytes, resulting in disruption of glycolysis and eventual cell death [5-8]. The progressive loss 
of chondrocytes results in histological and morphological changes to the articular cartilage, closely resembling those seen in human OA [9]. We have shown previously that joints contain mechanosensitive nerves [10] which become sensitized following MIA-induced joint degeneration [11,12] leading to chronic joint pain [13].

Voltage- gated sodium channels (VGSCs) are fundamental in regulating the excitability of neurons and overexpression of these channels can produce abnormal spontaneous firing patterns which underpin chronic pain $[14,15]$. There are at least nine different VGSC subtypes in the nervous system, and each subtype can be functionally classified as either tetrodotoxin-sensitive or tetrodotoxin-resistant [16]. Neuronal sodium channel subtypes including Nav1.3, Nav1.7, Nav1.8, and Nav1.9 have been implicated in the processing of nociceptive information [17]. The VGSC Nav1.8 is a tetrodotoxinresistant sodium channel with a distribution restricted to primary afferent neurons $[18,19]$ and the majority of Nav1.8-containing afferents transmit nociceptive signals to pain processing areas of the spinal cord [20]. Changes in the expression, trafficking and redistribution of Nav1.8 following inflammation or nerve injury are thought to be a major contributor to the sensitization of afferent nerves and the generation of pain [21]. The exclusive distribution of this channel on nociceptive neurons makes it an attractive target for the treatment of pain. Targeting of the Nav1.8 sodium channel with genetic deletions and antisense treatment has confirmed that this channel contributes to normal and pathological nociception [19,22-24]. A-803467 is a potent and selective Nav1.8 sodium channel blocker which has been found to attenuate pain in different rat models of neuropathic and inflammatory pain when given systemically [25-27]. The present study investigated if local intraarterial administration of A-803467 could reduce the hypersensitivity of nociceptive joint afferents and the resultant joint pain in the rat MIA model of OA.

\section{Materials and methods}

\section{Animals}

Experiments were performed on 44 male Wistar rats (340 to $430 \mathrm{~g}$ ) which were housed in cages at room temperature $\left(22^{\circ} \mathrm{C}\right)$ under a 12:12 hour light/dark cycle with free access to water and food. The animal handling and surgical procedures outlined in this study all received ethical approval from the University of Calgary Animal Care Committee and adhered to the Canadian Council for Animal Care guidelines for the care and use of experimental animals.

\section{Induction of $O A$}

Forty-four rats were deeply anaesthetised with $2 \%$ isoflurane in $100 \%$ O2 (1 L/minute) until the flexor withdrawal reflex was abolished. OA was induced by an injection of $3 \mathrm{mg}$ sodium monoiodoacetate (Tocris Bioscience, Missouri USA; $50 \mu \mathrm{l}$ volume) in $0.9 \%$ saline into the joint cavity through the patellar ligament. Animals were allowed to recover for 14 days which has consistently been shown to cause severe end-stage OA in this species $[5,7,8]$.

\section{Surgical preparation for electrophysiological recordings}

Following OA development, animals were deeply anaesthetized with urethane ( $25 \%$ stock solution; $2 \mathrm{~g} / \mathrm{kg}$, i.p.) and an acceptable depth of anaesthesia was determined by the absence of the hindpaw withdrawal reflex. Core body temperature was measured by a rectally inserted thermometer and maintained at $37^{\circ} \mathrm{C}$. The trachea was cannulated and connected to a Harvard rodent respiratory pump to allow artificial ventilation with $100 \% \mathrm{O} 2$ (stroke volume: $2.5 \mathrm{ml}$ breath frequency: 60 breaths/ min). The left carotid artery was then exposed and cannulated with a fine bore catheter (Portex Fine Bore Tubing, $0.5 \mathrm{~mm}$ ID, $1.00 \mathrm{~mm}$ OD; SIMS Portex Ltd., Kent, UK) containing heparinized saline (100 units $/ \mathrm{ml})$. The cannula was connected to a pressure transducer to allow continuous blood pressure measurement as recorded by a blood pressure monitor (BP-1, World Precision Instruments, Sarasota, FL, USA). An additional catheter containing heparinized saline was placed in the left jugular vein (Portex Fine Bore Tubing, $0.40 \mathrm{~mm}$ ID, $0.80 \mathrm{~mm}$ OD; SIMS Portex Ltd., Kent, UK) and a single administration of the muscle relaxant gallamine triethiodide (Sigma-Aldrich, Ontario, Canada; $50 \mathrm{mg} / \mathrm{kg}$ ) was injected to eliminate neural interference arising from the hindlimb musculature. The right saphenous artery was cannulated below the knee joint to permit local close intra-arterial injection of A-803467 to the knee joint. A specialized clamp was fixed to the mid-shaft of the isolated right femur and attached to a stereotaxic frame to prevent movement of the proximal aspect of the rat hindlimb. To apply rotation to the knee joint, the right hindpaw was placed in a shoe-like holder and the hindlimb rotated to standardized torque levels as measured by a force transducer and a torque meter (MVD2510; Hottinger-Baldwin Messtechnik, Darmstadt, Germany). Finally, a longitudinal skin incision was made along the medial aspect of the hindlimb and the skin flaps were fixed to a metal ' $\mathrm{O}$ ' ring to create a pouch which was filled with warm paraffin oil to prevent tissue desiccation throughout the experiment. The technique used for recording afferent activity from articular nerve fiber from rat knee joints has been described previously [28]. To prevent input from the foot and ankle region, the saphenous nerve was transected distally to the knee joint. The saphenous nerve was then isolated in the inguinal region and cut centrally to prevent the 
generation of spinally mediated reflexes. The saphenous nerve stump projecting from the knee was placed on a small, black Perspex stage. Under a dissecting microscope, the perineurium was removed and fine neurofilaments were dissected free from the nerve using fine watchmaker forceps. The neural elements were then placed over a platinum electrode to record single afferent fiber activity. To ensure that recorded fibers originate from the knee joint, the receptive field of the fibers was identified by the elicitation of a response to gentle probing of the knee joint with a glass rod with a $1 \mathrm{~mm}$ tip. The mechanical threshold of each recorded joint afferent was determined by a gradual increase of torque to the joint until the fiber started to elicit action potentials. Non-noxious and noxious outward movements were then applied to the knee with each movement lasting ten seconds. Non-noxious movement is defined here as being within the normal working range of the joint, while noxious rotation refers to torque occurring outside the normal range but not severe enough to cause soft tissue injury. After three movement cycles, identical test rotations were repeated every two minutes such that each fiber was subjected to a total of 22 movements. The conduction velocity of the nerve fibers (C-fibers $<2$ meters/second; A $\delta$-fibers 2-10 meters/second) was determined by electrical stimulation of the receptive field with a bipolar silver wire electrode $(1-10 \mathrm{~V}, 0.5 \mathrm{msec}$ pulse width). The conduction velocity was calculated by dividing the distance between the receptive field and the electrode by the latency between the stimulus artefact and the evoked action potential. Recordings were made before (control) and after close intra-arterial injection of the Nav 1.8 channel blocker A-803467. The dose of A-803467 administered was 500 ug in 100 ul vehicle (2\% dimethyl sulfoxide (DMSO), $1 \%$ cremophor, $0.9 \%$ saline). This dose has previously been shown to be effective in attenuating evoked pain in inflamed hindpaws [27]. Percentage changes in nerve firing rate before and after administration of A-803467 were calculated. Neuronal activity was recorded by a data acquisition system (CED1401, Cambridge Electronic Design, Cambridge, UK) and stored on a microcomputer for off-line analysis. The number of action potentials/movement was determined using Spike 2 software (Cambridge Electronic Design, Cambridge, UK).

\section{Behavioral assessment of pain}

For measuring joint pain behavior, MIA-treated rats were regularly handled and gradually habituated to the test equipment on three consecutive days prior to behavioral testing. Hindlimb weight bearing was determined using an incapacitance tester (Linton, Norfolk, UK) consisting of a dual channel weight averager. The force exerted by each hindlimb (measured in grams) was averaged over a five second period. Each data point is the mean of three consecutive readings. For MIA animals, weight distribution was measured between treated (intra-articular injection of A$80346710 \mathrm{mg} ; 100 \mu \mathrm{l}$ bolus) and contralateral non-treated hindlimbs. For secondary allodynia measurements, a von Frey hair algesiometer (Ugo-Basile, Milan, Italy) was employed to determine the force required to elicit a withdrawal response to a tactile mechanical stimulus applied to the hindpaw. The rat was placed on a metal mesh surface in an enclosed area and allowed to move about freely. With the animal at rest (that is, in the absence of any exploratory or grooming behavior) a touch stimulator unit was oriented under the animal and using an adjustable angled mirror the stimulating microfilament $(0.5 \mathrm{~mm}$ diameter) was positioned below the plantar surface of the paw. Activation of the unit caused the fine metal monofilament to advance with constant speed and touch the paw in the proximal metatarsal region. The filament exerts a gradually increasing force to the plantar surface, starting below the threshold of detection and increasing until the stimulus becomes painful and the rat withdraws its paw. The force required to elicit a paw withdrawal reflex is automatically recorded and measured in grams. A maximum force of $50 \mathrm{~g}$ and a ramp speed of $4.5 \mathrm{~g} /$ second were chosen for all of the algesiometry tests.

\section{Drugs and reagents}

Sodium monoiodoacetate and A-803467 were obtained from Tocris Bioscience (Minneapolis, MN, USA; Bristol, UK); gallamine triethiodide, DMSO, cremophor and urethane were obtained from Sigma Aldrich (Oakville, Ontario, Canada; Poole, UK). A-803467 was dissolved in vehicle solution ( $2 \% \mathrm{DMSO}, 1 \%$ cremophor, $0.9 \%$ saline) and aliquots of the drug were kept frozen $\left(-20^{\circ} \mathrm{C}\right)$ in Eppendorf vials until required. The $\mathrm{pH}$ of all solutions was determined to exclude acidity as a sensitizing factor on afferent nerve fibers. All solutions were found to have a neutral $\mathrm{pH}(\mathrm{pH}$ 7.4) before injection. Gallamine triethiodide was made fresh on the day of experimentation and dissolved in $0.9 \%$ saline.

\section{Statistics}

All data were normally distributed and expressed as means \pm SEM for ' $n$ ' observations. Establishment of joint pain and secondary allodynia after MIA injection was confirmed using a Student's t-test. The effect of A803467 on nociceptive activity was analyzed by two-way analysis of variance (ANOVA) with a Bonferroni post test. All differences were considered statistically significant when $P$ was less than 0.05 .

\section{Results}

\section{Electrophysiological recordings}

Between one and three afferent fibers were examined per animal such that a total of 19 units were recorded in this study. All fibers responded to noxious stimuli 
while only six units responded in both the normal and noxious working range. A wash out period of at least 60 minutes was observed between administration of A803467 to multiple fibers in the same animal. In case the firing rate of a recorded afferent did not return to baseline level after 30 minutes no further afferents were recorded in that particular animal. The conduction velocities of recorded afferents ranged from 0.57 to $2.87 \mathrm{~m} /$ s. Approximately $20 \%$ of the recorded fibers were classified as A $\delta$ fibers with the remaining fibers being slowly conducting $\mathrm{C}$-fibers. The mechanical threshold of the recorded fibers ranged from 5 to $35 \mathrm{mNm}$ The average firing rate of afferents in the MIA-injected joints during non-noxious joint rotation was $43 \pm 6.21$ action potentials/10 s movement $(n=10)$ and $114 \pm 9.95$ action potentials $/ 10 \mathrm{~s}$ movement with noxious joint rotation $(n$ =16) These firing rates are consistent with previous studies that showed a significant increase in firing rate in MIA-treated rats compared to saline injected control joints [12].

\section{Effect of A-803467 on joint afferent mechanosensitivity}

With non-noxious rotation of the MIA-injected rat knee joint, local administration of A-803467 had no effect on mechanosensitivity of joint afferents; however, A-803467 reduced the mechanosensitivity during noxious rotation of the joint. A specimen recording from a typical joint afferent before and after either vehicle or A-803467 application is shown in Figure $1 \mathrm{~A}$ and $1 \mathrm{~B}$ respectively. The desensitizing effect of A-803467 during noxious joint rotation was significant at 11 minutes after drug application and reached a maximum at the 13 minute time point (mean: $-51.45 \% \pm 8.81$; \% change compared to control). Compared to vehicle, A-803467 had no significant effect on firing rate during non-noxious rotation $(P=0.49$ two-way ANOVA with Bonferroni's post-test; $n=6 /$ group (Figure $2 \mathrm{~A}$ ); but significantly reduced the firing rate during noxious joint rotation $(P<0.0001$ two-way ANOVA with Bonferroni 's post-test; $n=10$ to 12 vehicle group, $n=18$ to 19 A-803467 group; Figure $2 \mathrm{~B})$. Both, $\mathrm{A}-\delta$ fibers and $\mathrm{C}$-fibers were equally responsive to A-803467. The vehicle was found to have no significant effect on joint mechanosensitivity. Spontaneous activity was observed in four of the recorded joint afferents. The mean frequency of spontaneous firing before drug application was $0.53 \pm 0.1 \mathrm{~Hz}$ and 1 minute after A-803467 afferent activity was $0.63 \pm 0.2 \mathrm{~Hz}$. The lack of effect of A-803467 on spontaneous activity was consistent across the 15 minute time course perineurium ( $P$ = 0.46 two-way ANOVA; $n=4$ /group; Figure 3).

Effect of A-803467 on joint pain and secondary allodynia The induction of OA caused a significant reduction in the amount of weight being borne on the ipsilateral

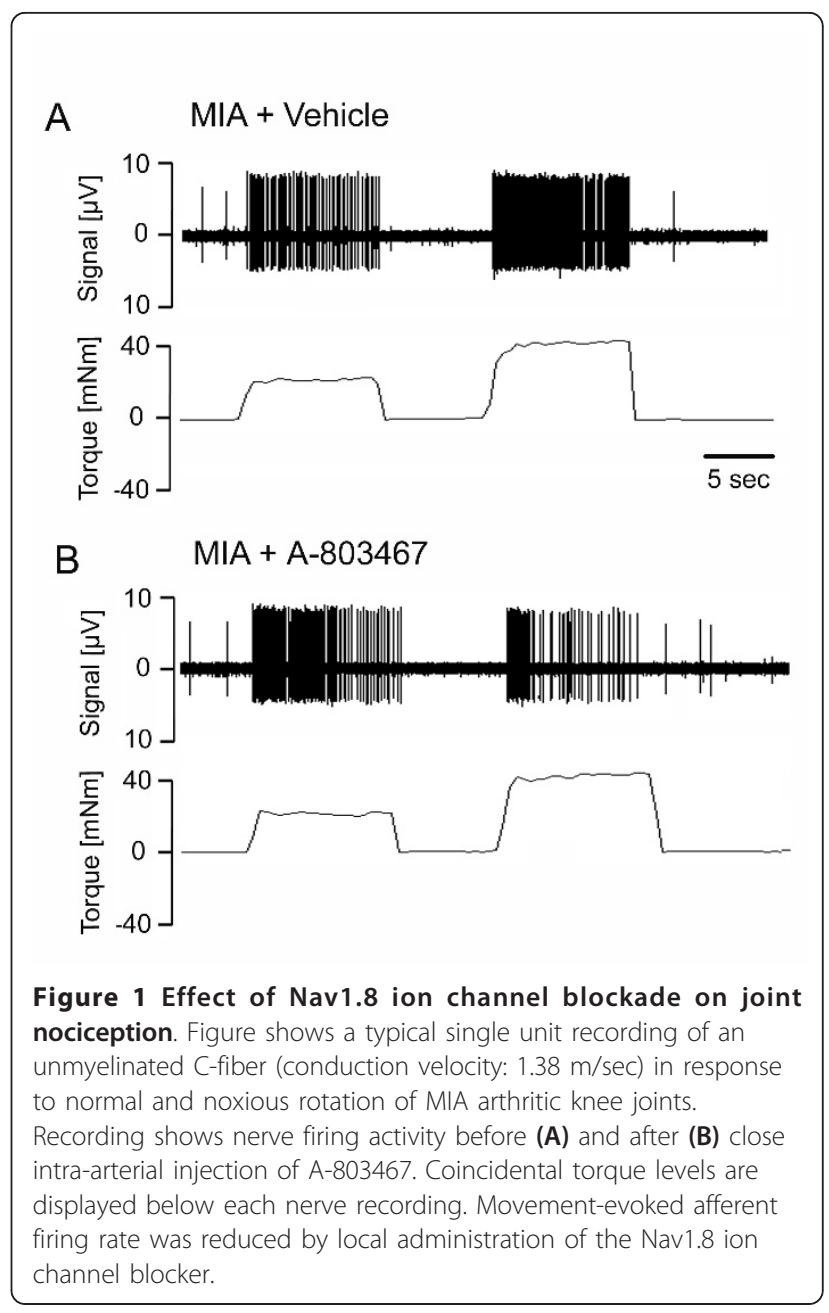

hindlimb compared to naïve rats $(P<0.0001$; unpaired Student's t-test; $n=20$ naïve rats, $n=6$ OA rats; Figure 4A). While naive rats distribute their body weight evenly between the two hindlimbs, in the MIA rat the weight placed on the ipsilateral hindlimb was reduced to about $35 \% \pm 1.46$. Intraarticular injection of A-803467 into the MIA-treated joint reduced the difference in weight bearing significantly such that $43 \% \pm 2.89$ of the animal's body weight was now placed on the ipsilateral hindlimb. This improvement in weight distribution was significant compared to vehicle treated animals $(P<0.0001$; twoway ANOVA with Bonferroni 's post test; $n=10$; Figure 4A) indicating that A-803467 reduced pain in MIA joints. Injection of the vehicle by itself had no effect on weight distribution.

Paw withdrawal threshold to mechanical stimuli in naïve animals (mean: $39.7 \mathrm{~g} \pm 1.69$ ), was significantly reduced in MIA arthritic rats (mean: $26.80 \mathrm{~g} \pm 1.27$ indicating the presence of secondary allodynia $(P=$ 0.0002; unpaired Student's t test; $n=10$ naïve rats, $n=$ 6 OA rats; Figure 4B). Intra-articular A-803467 injection 
A

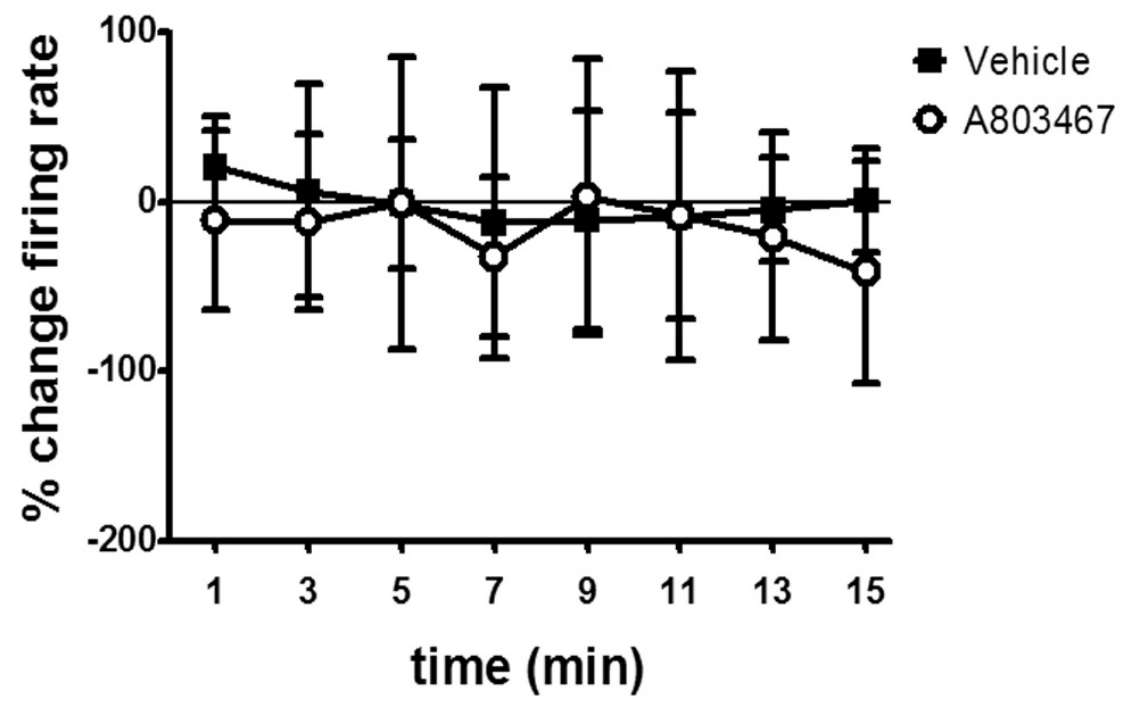

B

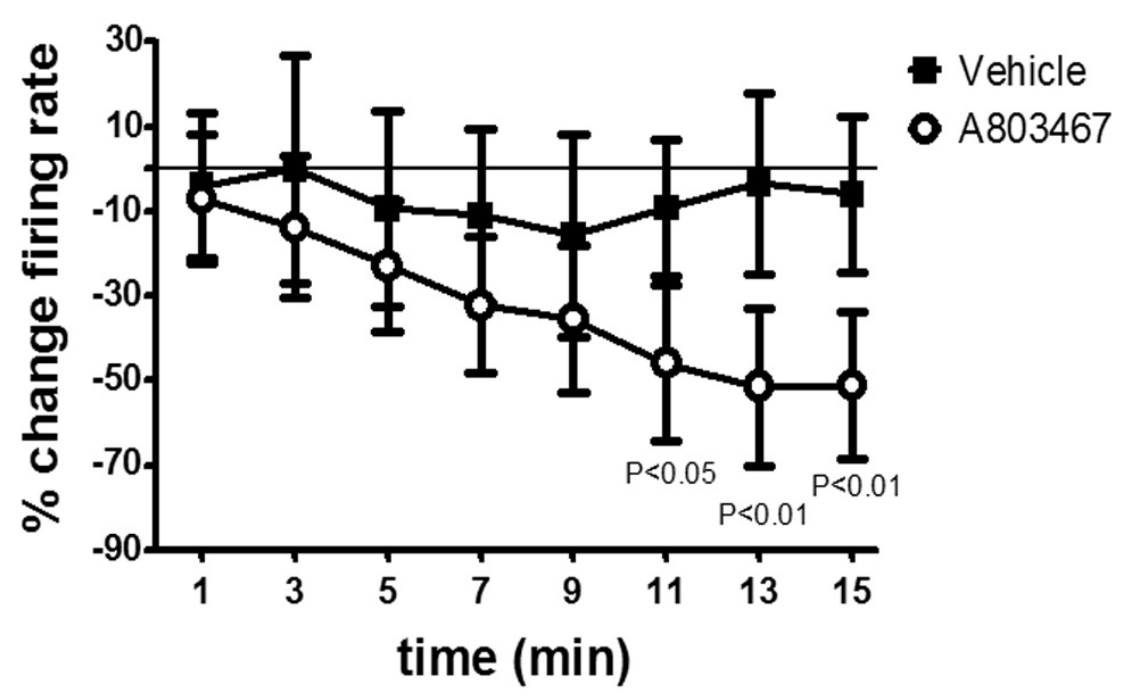

Figure 2 Time course of effect of A-803467 on joint mechanosensitivity. Local application of A-803467 had no effect on joint afferent firing rate during non noxious rotation of the joint $(\mathbf{A}) n=6 /$ group, but significantly reduced nociceptor activity during noxious joint rotation (B). 2way ANOVA; $n=10$ to 12 vehicle group, $n=18$ to 19 A-803467 group. Values are means \pm SEM. 


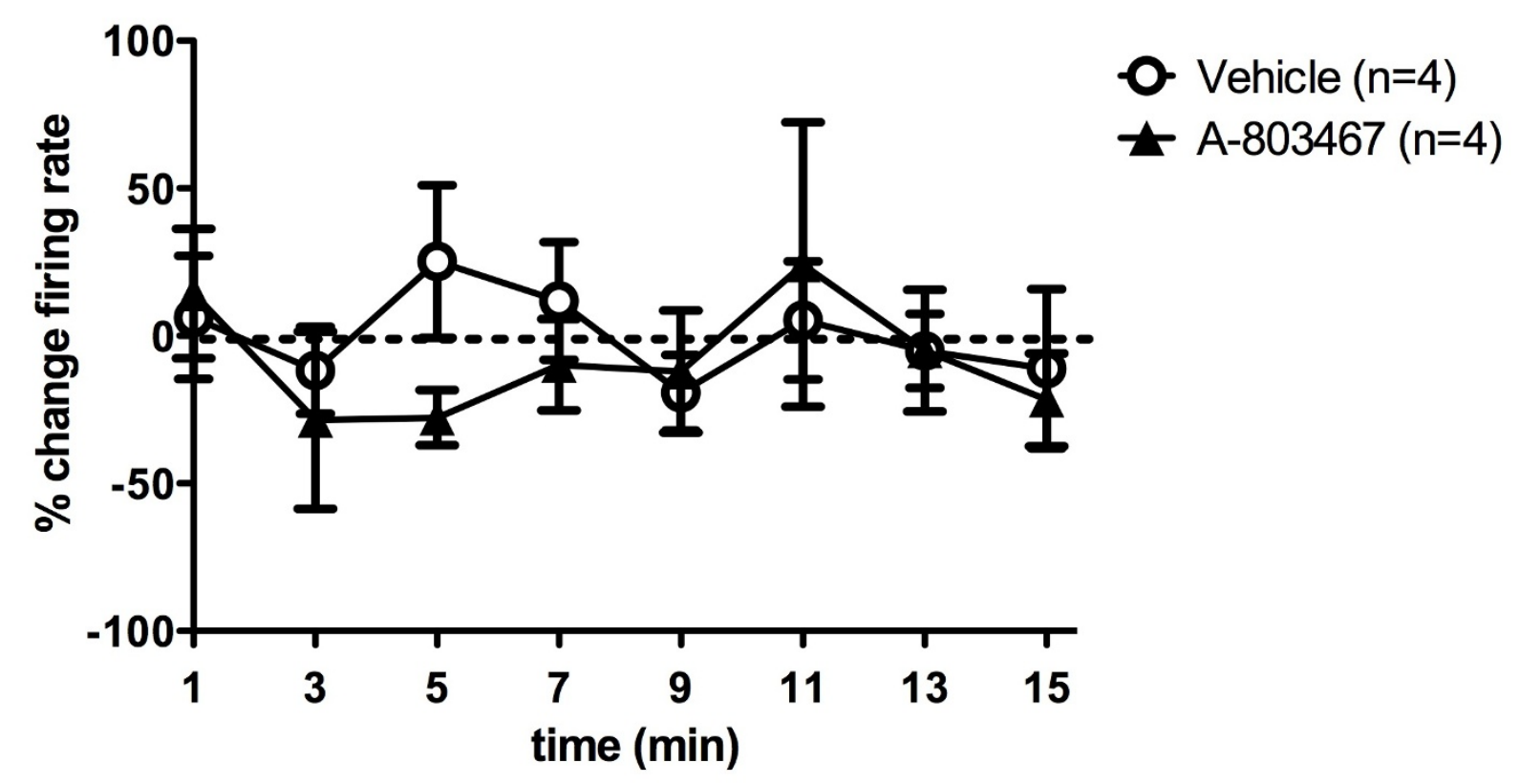

Figure 3 Effect of A-803467 on joint afferent spontaneous activity. Compared to vehicle, local injection of A-803467 had no effect on nerve spontaneous activity ( $n=4 /$ group). Values are means \pm SEM.

significantly reduced this MIA-induced mechanical hypersensitivity (mean: $32.25 \mathrm{~g} \pm 1.84$ ) compared to vehicle treated animals (mean: $22.83 \mathrm{~g} \pm 2.62$; $P<$ 0.0001, two-way ANOVA with Bonferroni's post test; $n$ $=10$; Figure 4B.

\section{Discussion}

Management of osteoarthritis pain remains a huge challenge in the clinic with millions of arthritis patients living with chronic disability. Limitations to current pharmacological treatment strategies include a lack of therapeutic efficacy and potentially hazardous sideeffects. Therefore, there is a pressing need to understand the underlying mechanisms that evoke OA pain and to develop new, more effective analgesics. One intuitive approach would be to attenuate pathological OAinduced pain by selectively blocking joint nociceptors while preserving other desirable physiological functions of the nerve. Intra-articular injection of non-selective sodium channel blockers has been shown to be effective for the treatment of OA pain [29] while intravenous administration of these agents can produce long lasting pain relief in both animal models [30] and in patients with intractable neuropathic pain [31]. The clear disadvantages of using non-selective sodium channel blockers locally are complete loss of sensation and disruption of normal physiological functions such as proprioception and neurovasoregulation. Systemic use of such blockers can lead to cardiotoxicity, sedation and cognitive impairment [32]. Targeting sodium channels which are located exclusively on nociceptive afferents could reduce these undesirable side effects. The novel small molecule A803467 selectively blocks the $\mathrm{Na}_{\mathrm{v}} 1.8$ sodium channel in vitro and in vivo [25]. The agent has been shown to inhibit inward currents in cultured rat dorsal root ganglia cells and $\mathrm{Na}_{\mathrm{v}} 1.8$ expressing HEK cells (IC50 = 8 nM). A-803467 was also found to reduce mechanicallyevoked firing of dorsal horn wide dynamic neurons as well as mechanical allodynia in animal models of inflammation and neuropathic pain over the dose range 10 to $100 \mathrm{mg} / \mathrm{kg}$. In this study it was found that local administration of A-803467 significantly reduced OA joint mechanosensitivity during noxious but not during nonnoxious rotation of the joint. These data confirm that blocking the Nav 1.8 channel attenuates joint mechanonociception in the MIA model of joint degeneration. The fact that the reduction in mechanosensitivity was limited to noxious joint rotation confirms that Nav1.8 plays a major role in transmitting strong noxious signals but does not affect physiological mechanosensitivity during normal, non-noxious rotation of the joint.

Approximately $50 \%$ of C-fibers and $10 \%$ of A- $\delta$ fibers in naive rats express Nav1.8 channels [33] and this proportion increases in painful conditions $[21,34]$. In 

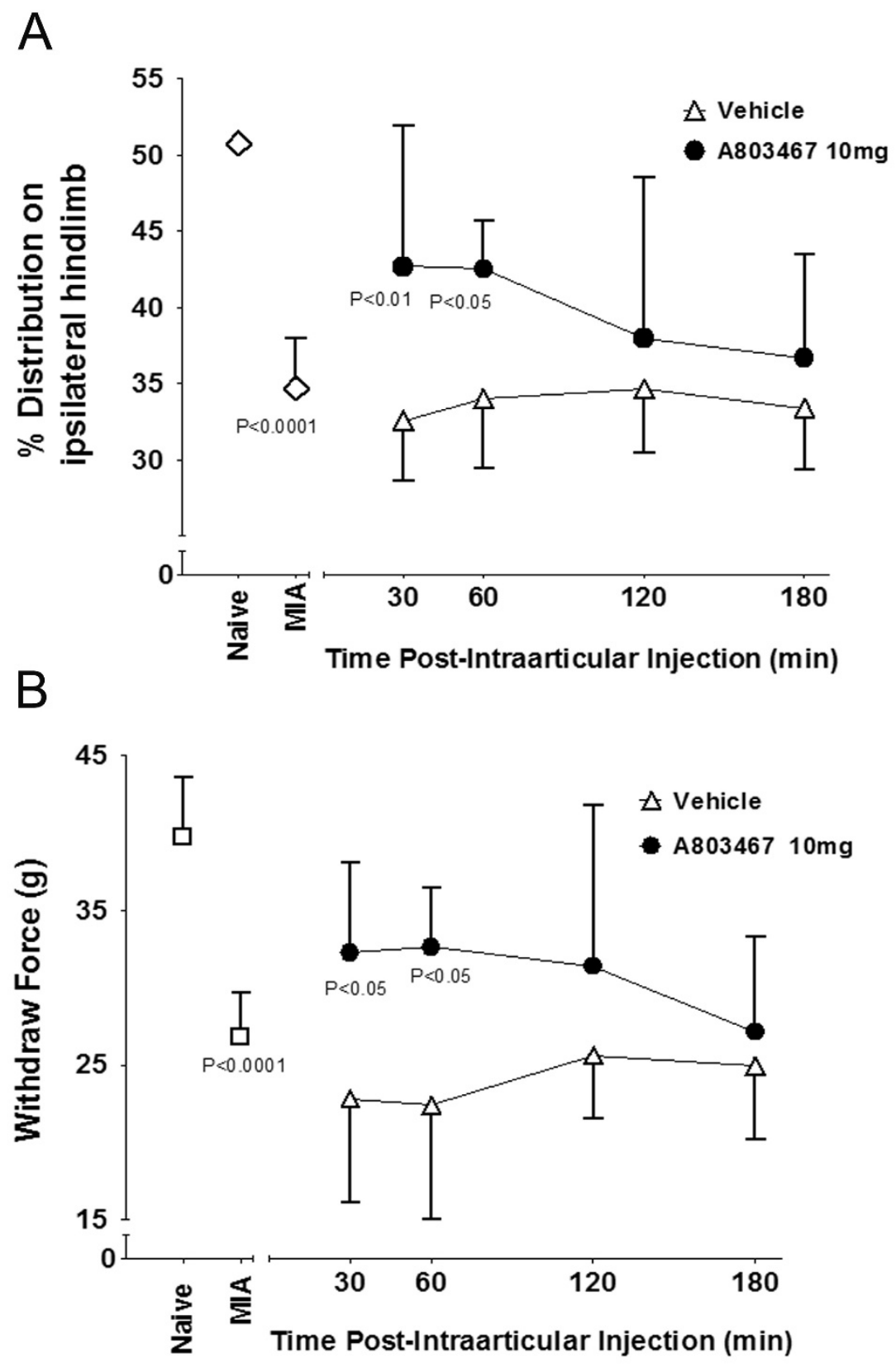

Figure 4 Pain behavioral responses to treatment with A-803467. In (A), MIA causes a profound shift in hindlimb weight distribution onto the unaffected hindlimb. Following A-803467 treatment body weight was re-distributed onto the MIA-treated joint reflecting an analgesic effect. In (B), hindpaw withdrawal thresholds were measured in response to an intra-articular injection of A-803467. The force required to elicit hindpaw withdrawal following A-803467 was significantly reduced compared to vehicle-treated animals, confirming a reduction in MIA induced secondary allodynia. Values are means \pm SEM. 
addition, a dynamic redistribution of Nav1.8 channels from dorsal root ganglia somata to the axons has been observed so that more Nav1.8 channels are expressed on $\mathrm{C}$-fibers and A- $\delta$ fibers in rat digital nerves after neuropathic injury [35]. In this study, the firing rate in about $75 \%$ of all recorded afferents was substantially reduced during noxious rotation after A-803467 administration. The relatively high percentage of responsive fibers to A-803467 in this study could be explained by similar channel reorganization and up-regulation in response to MIA induced joint pathology. Systemic administration of A-803467 has been shown to reduce pain significantly in several models of neuropathic and inflammatory pain [25-27] while preserving motor function as assessed by spontaneous exploratory behavior, and motor coordination as assessed by the rotarod and edge test assays [25]. In this study, an intra-articular injection of A-803467 significantly reduced hindlimb incapacitance and secondary allodynia in MIA-treated rats. This observation corroborates the electrophysiological data which signified an anti-nociceptive effect of the channel blocker in diseased joints. The inhibitory effect of intra-articular A-803467 on hindlimb incapacitance confirms that joint Nav1.8 channels are involved in OA pain and that peripheral modulation of these channels has analgesic potential. The ability of intraarticular A-803467 to reduce secondary allodynia in this study is also of importance as it suggests that the referred pain associated with OA can be moderated by blocking Nav1.8 channels in the knee.

Although the evidence presented here highlights the involvement of peripheral Nav1.8 channels in producing OA pain, Nav1.8 channels present on the central terminals of primary afferent nerves also need to be considered. It has recently been demonstrated that A-803467 has good penetration into the central nervous system where it produces a more robust antinociception in neuropathic pain models than a peripherally acting nonselective sodium channel blocker [36]. This ability of A803467 to suppress spinal dorsal horn neuronal excitability and to reduce allodynia effectively after nerve injury provides further evidence that inhibition of Nav1.8 channels on peripheral nerves, with synaptic connections in the spinal cord, is an important site of nociceptive sensory processing [37] and could be necessary to achieve more pronounced and longer lasting analgesia.

The present investigation also found that A-803467 had no significant effect on spontaneous nerve activity in MIA-treated joints. The reason for the lack of effect of peripheral A-803467 administration on MIA-induced spontaneous activity could be that articular Nav1.8 channels are only activated by external physical stimuli and are unaffected by the sensitizing effect of algogenic agents present in the diseased joint. The central terminal of the primary afferent neuron, however, could still be a useful target for reducing spontaneous activity and could therefore play a role in the reduction of OA pain at rest. This hypothesis is supported by studies showing that intrathecal administration of A-803467 significantly reduced evoked and spontaneous firing of wide dynamic range neurons [25] whereas local intra-plantar application only reduced evoked but not spontaneous nerve firing [27]. Since spontaneous activity is necessary for the perception of chronic, on-going pain in MIA joints [11], intrathecal A-803467 may be required to address the problem of pain at rest in OA patients.

In conclusion, this study shows for the first time that the Nav1.8 channel is involved in mediating nociception and pain in a rat model of OA. We propose that targeting Nav1.8 channels could be a promising approach to reduce nociceptor sensitivity and consequently pain perception in OA while avoiding the undesirable sideeffects of non-selective sodium channel blockers.

\section{Abbreviations}

ANOVA: analysis of variance; MIA: monoiodoacetate; OA: osteoarthritis; VGSC: voltage gated sodium channel.

\section{Acknowledgements}

We thank Dr. Shu Zhan for her technical assistance. This work was supported by a grant from the Canadian Institutes for Health Research. $J J M C D$ receives salary support from the Alberta Heritage Foundation for Medical Research (AHFMR) and The Arthritis Society of Canada. NS is an AHFMR and Canadian Arthritis Network Postdoctoral Fellow.

\section{Author details}

'Department of Physiology \& Pharmacology, University of Calgary, 3330, Hospital Drive NW, Calgary, AB, T2N 4N1, Canada. ${ }^{2}$ Departments of Pharmacology \& Anaesthesia, Dalhousie University, 5850, College Street, PO Box 15000, Halifax, NS B3H 4R2, Canada.

\section{Authors' contributions}

NS helped design the experiments, carried out the electrophysiology, helped analyze the data and contributed to writing the manuscript. JJMCD helped design the experiments, assisted in data analysis and helped write the manuscript. Both authors read and approved the final manuscript.

\section{Competing interests}

The authors declare that they have no competing interests.

Received: 21 September 2011 Revised: 16 December 2011

Accepted: 7 January 2012 Published: 7 January 2012

\section{References}

1. Coggeshall RE, Hong KA, Langford LA, Schaible HG, Schmidt RF: Discharge characteristics of fine medial articular afferents at rest and during passive movements of inflamed knee joints. Brain Res 1983, 272:185-188

2. McDougall JJ: Pain and OA. J Musculoskelet Neuronal Interact 2006, 6:385-386.

3. McDougall JJ: Arthritis and pain. Neurogenic origin of joint pain. Arthritis Res Ther 2006, 8:220.

4. Schaible HG, Grubb BD: Afferent and spinal mechanisms of joint pain. Pain 1993, 55:5-54.

5. Bove SE, Calcaterra SL, Brooker RM, Huber CM, Guzman RE, Juneau PL, Schrier DJ, Kilgore KS: Weight bearing as a measure of disease progression and efficacy of anti-inflammatory compounds in a model of 
monosodium iodoacetate-induced osteoarthritis. Osteoarthritis Cartilage 2003, 11:821-830.

6. Fernihough J, Gentry C, Malcangio M, Fox A, Rediske J, Pellas T, Kidd B, Bevan S, Winter J: Pain related behaviour in two models of osteoarthritis in the rat knee. Pain 2004, 112:83-93.

7. Guingamp C, Gegout-Pottie P, Philippe L, Terlain B, Netter P, Gillet P: Monoiodoacetate-induced experimental osteoarthritis: a dose-response study of loss of mobility, morphology, and biochemistry. Arthritis Rheum 1997, 40:1670-1679.

8. Kalbhen DA: Chemical model of osteoarthritis-a pharmacological evaluation. J Rheumatol 1987, 14 Spec No:130-131.

9. Janusz MJ, Hookfin EB, Heitmeyer SA, Woessner JF, Freemont AJ, Hoyland JA, Brown KK, Hsieh LC, Almstead NG, De B, Natchus MG, Pikul S, Taiwo YO: Moderation of iodoacetate-induced experimental osteoarthritis in rats by matrix metalloproteinase inhibitors. Osteoarthritis Cartilage 2001, 9:751-760.

10. Heppelmann B, McDougall JJ: Inhibitory effect of amiloride and gadolinium on fine afferent nerves in the rat knee: evidence of mechanogated ion channels in joints. Exp Brain Res 2005, 167:114-118.

11. Schuelert N, McDougall JJ: Electrophysiological evidence that the vasoactive intestinal peptide receptor antagonist VIP6-28 reduces nociception in an animal model of osteoarthritis. Osteoarthritis Cartilage 2006, 14:1155-1162.

12. Schuelert N, McDougall Jj: Grading of monosodium iodoacetate-induced osteoarthritis reveals a concentration-dependent sensitization of nociceptors in the knee joint of the rat. Neurosci Lett 2009, 465:184-188.

13. Liu P, Okun A, Ren J, Guo RC, Ossipov MH, Xie J, King T, Porreca F: Ongoing pain in the MIA model of osteoarthritis. Neurosci Lett 2011, 493:72-75.

14. Lai J, Porreca F, Hunter JC, Gold MS: Voltage-gated sodium channels and hyperalgesia. Annu Rev Pharmacol Toxicol 2004, 44:371-397.

15. Waxman SG, Cummins TR, Dib-Hajj S, Fjell J, Black JA: Sodium channels, excitability of primary sensory neurons, and the molecular basis of pain. Muscle Nerve 1999, 22:1177-1187.

16. Wood JN, Boorman JP, Okuse K, Baker MD: Voltage-gated sodium channels and pain pathways. J Neurobiol 2004, 61:55-71.

17. Amir R, Argoff CE, Bennett GJ, Cummins TR, Durieux ME, Gerner P, Gold MS, Porreca F, Strichartz GR: The role of sodium channels in chronic inflammatory and neuropathic pain. J Pain 2006, 7:S1-29.

18. Akopian AN, Sivilotti L, Wood JN: A tetrodotoxin-resistant voltage-gated sodium channel expressed by sensory neurons. Nature 1996, 379:257-262.

19. Akopian AN, Souslova V, England S, Okuse K, Ogata N, Ure J, Smith A, Kerr BJ, McMahon SB, Boyce S, Hill R, Stanfa LC, Dickenson AH, Wood JN: The tetrodotoxin-resistant sodium channel SNS has a specialized function in pain pathways. Nat Neurosci 1999, 2:541-548

20. Djouhri L, Fang X, Okuse K, Wood JN, Berry CM, Lawson SN: The TTXresistant sodium channel Nav1.8 (SNS/PN3): expression and correlation with membrane properties in rat nociceptive primary afferent neurons. $J$ Physiol 2003, 550:739-752.

21. Strickland IT, Martindale JC, Woodhams PL, Reeve AJ, Chessell IP, McQueen DS: Changes in the expression of NaV1.7, NaV1.8 and NaV1.9 in a distinct population of dorsal root ganglia innervating the rat knee joint in a model of chronic inflammatory joint pain. Eur J Pain 2008, 12:564-572.

22. Joshi SK, Mikusa JP, Hernandez G, Baker S, Shieh CC, Neelands T, Zhang XF, Niforatos W, Kage K, Han P, Krafte D, Faltynek C, Sullivan JP, Jarvis MF, Honore P: Involvement of the TTX-resistant sodium channel Nav 1.8 in inflammatory and neuropathic, but not post-operative, pain states. Pain 2006, 123:75-82.

23. Lai J, Gold MS, Kim CS, Bian D, Ossipov MH, Hunter JC, Porreca F: Inhibition of neuropathic pain by decreased expression of the tetrodotoxinresistant sodium channel, NaV1.8. Pain 2002, 95:143-152.

24. Porreca F, Lai J, Bian D, Wegert S, Ossipov MH, Eglen RM, Kassotakis L, Novakovic S, Rabert DK, Sangameswaran L, Hunter JC: A comparison of the potential role of the tetrodotoxin-insensitive sodium channels, PN3/SNS and NaN/SNS2, in rat models of chronic pain. Proc Natl Acad Sci USA 1999, 96:7640-7644.

25. Jarvis MF, Honore P, Shieh CC, Chapman M, Joshi S, Zhang XF, Kort M, Carroll W, Marron B, Atkinson R, Thomas J, Liu D, Krambis M, Liu Y, McGaraughty S, Chu K, Roeloffs R, Zhong C, Mikusa JP, Hernandez G, Gauvin D, Wade C, Zhu C, Pai M, Scanio M, Shi L, Drizin I, Gregg R,
Matulenko M, Hakeem A, et al: A-803467, a potent and selective Nav1.8 sodium channel blocker, attenuates neuropathic and inflammatory pain in the rat. Proc Natl Acad Sci USA 2007, 104:8520-8525.

26. Joshi SK, Honore P, Hernandez G, Schmidt R, Gomtsyan A, Scanio M, Kort M, Jarvis MF: Additive antinociceptive effects of the selective Nav1.8 blocker A-803467 and selective TRPV1 antagonists in rat inflammatory and neuropathic pain models. J Pain 2009, 10:306-315.

27. McGaraughty S, Chu KL, Scanio MJ, Kort ME, Faltynek CR, Jarvis MF: A selective Nav1.8 sodium channel blocker, A-803467 [5-(4-chlorophenyl$\mathrm{N}$-(3,5-dimethoxyphenyl)furan-2-carboxamide], attenuates spinal neuronal activity in neuropathic rats. J Pharmacol Exp Ther 2008, 324:1204-1211

28. McDougall JJ, Hanesch U, Pawlak M, Schmidt RF: Participation of NK1 receptors in nociceptin-induced modulation of rat knee joint mechanosensitivity. Exp Brain Res 2001, 137:249-253.

29. Karaman Y, Kayali C, Ozturk H, Kaya A, Bor C: A comparison of analgesic effect of intra-articular levobupivacaine with bupivacaine following knee arthroscopy. Saudi Med J 2009, 30:629-632.

30. Araujo MC, Sinnott CJ, Strichartz GR: Multiple phases of relief from experimental mechanical allodynia by systemic lidocaine: responses to early and late infusions. Pain 2003, 103:21-29.

31. Kastrup J, Petersen P, Dejgard A, Angelo HR, Hilsted J: Intravenous lidocaine infusion-a new treatment of chronic painful diabetic neuropathy? Pain 1987, 28:69-75

32. Kinloch RA, Cox PJ: New targets for neuropathic pain therapeutics. Expert Opin Ther Targets 2005, 9:685-698.

33. Amaya F, Decosterd I, Samad TA, Plumpton C, Tate S, Mannion R, Costigan M, Woolf $\mathrm{CJ}$ : Diversity of expression of the sensory neuronspecific TTX-resistant voltage-gated sodium ion channels SNS and SNS2. Mol Cell Neurosci 2000, 15:331-342.

34. Coggeshall RE, Tate S, Carlton SM: Differential expression of tetrodotoxinresistant sodium channels Nav1.8 and Nav1.9 in normal and inflamed rats. Neurosci Lett 2004, 355:45-48

35. Novakovic SD, Tzoumaka E, McGivern JG, Haraguchi M, Sangameswaran L, Gogas KR, Eglen RM, Hunter JC: Distribution of the tetrodotoxin-resistant sodium channel PN3 in rat sensory neurons in normal and neuropathic conditions. J Neurosci 1998, 18:2174-2187.

36. Brochu RM, Dick IE, Tarpley JW, McGowan E, Gunner D, Herrington J, Shao PP, Ok D, Li C, Parsons WH, Stump GL, Regan CP, Lynch JJ Jr, Lyons KA, McManus OB, Clark S, Ali Z, Kaczorowski GJ, Martin WJ, Priest BT: Block of peripheral nerve sodium channels selectively inhibits features of neuropathic pain in rats. Mol Pharmacol 2006, 69:823-832.

37. Gu JG, MacDermott AB: Activation of ATP P2X receptors elicits glutamate release from sensory neuron synapses. Nature 1997, 389:749-753.

doi:10.1186/ar3553

Cite this article as: Schuelert and McDougall: Involvement of Nav 1.8 sodium ion channels in the transduction of mechanical pain in a rodent model of osteoarthritis. Arthritis Research \& Therapy 2012 14:R5.

\section{Submit your next manuscript to BioMed Central and take full advantage of:}

- Convenient online submission

- Thorough peer review

- No space constraints or color figure charges

- Immediate publication on acceptance

- Inclusion in PubMed, CAS, Scopus and Google Scholar

- Research which is freely available for redistribution

Submit your manuscript at www.biomedcentral.com/submit
C Biomed Central 